\title{
Shocked, breathless, and bloodied: Point-of-care ultrasound on the front line
}

\author{
Paul R. Atkinson, MB BCh BAO, MA*; D. Lewis, MBBS*
}

Point-of-care ultrasound (PoCUS) has come a long way since the days when ultrasound was a hidden dark art, only practised deep inside the recesses of radiology departments. The transition of the use of this technology from the assessment of relatively stable patients, into a tool used by front line clinicians has been proceeded steadily, to the point where many now consider PoCUS to be standard of care, much like the stethoscope, when assessing patients clinically. ${ }^{1}$ In this issue of CFEM, three groups of authors tackle questions related to the use of PoCUS on the front line of emergency medicine - in the resuscitation room.

The use of PoCUS in undifferentiated hypotensive, or shocked, patients has evolved from the targeting of specific individual pathologies and etiologies, to the development of systematized PoCUS protocols designed to answer physiological questions, identify pathology, and guide resuscitation. ${ }^{2-4}$ Stickles et al. looked at how reliable one of the most popular and commonly used of these protocols, the Rapid Ultrasound for Shock and Hypotension (RUSH) protocol, is in terms of accurately identifying the underlying category of shock in hypotensive patients. ${ }^{4,5}$ Jones et al. previously reported that early use of a similar PoCUS protocol helped narrow the differential diagnosis when compared with delayed use, in one of the first comparative studies of PoCUS. ${ }^{2}$ In their analysis, Stickles et al. performed a systematic review and meta-analysis of the literature, focusing on diagnostic studies for RUSH. ${ }^{5}$ This well-done analysis included four relevant studies totalling 357 patients. With sensitivities ranging from $64 \%$ for distributive, to $93 \%$ for obstructive shock, and specificities ranging from $80 \%$ for mixed, to $98 \%$ for obstructive, they concluded that when used in isolation, the RUSH exam is imperfect, particularly in excluding any subcategory of shock other than obstructive, potentially leading to an incomplete or incorrect diagnosis. In keeping with early teaching on all forms of PoCUS, this study confirms RUSH as being more valuable as a "rule-in" rather than a "rule-out" test, and importantly that this type of PoCUS protocol is best used as one component in the complete evaluation of critically ill patients, rather than the sole determinant for decision-making. We agree with the authors when they call for a more evidence-based approach to the use of PoCUS in this population and other patient groups. Rather than continuing to create more versions of what are essentially very similar lists of scans, combined as differently named protocols, we agree that the approach to PoCUS in shock should be based upon identifying the likely underlying etiology, answering immediate clinical questions, and integrating findings into clinical decisionmaking alongside other sources of information relating to the patient. ${ }^{6}$ We also agree that further comparative research is required to compare various approaches to PoCUS in critical illness with each other and with non-PoCUS-based approaches. ${ }^{7,8}$ The authors do not provide any information on comparisons with standard non-PoCUS-based care. Although PoCUS may have reasonable diagnostic performance characteristics in shock, does it perform better than standard care, in general, or for the detection of key etiologies?

Another group of critically ill patients seen on the front line are elderly patients with undifferentiated breathlessness. When the clinical evaluation fails to clarify the underlying etiology, tools such as PoCUS, portable chest radiography, and lab tests such as Brain natriuretic peptide $(\mathrm{BNP})$ can guide the clinician with

From the *Department of Emergency Medicine, Dalhousie University, Saint John Regional Hospital, Saint John, NB.

Correspondence to: Dr. Paul Atkinson, Department of Emergency Medicine, 400 University Ave., Saint John Regional Hospital, Saint John, NB E2L 4L4; Email: paul.atkinson@dal.ca

(c) Canadian Association of Emergency Physicians

CJEM 2019;21(3):321-323

DOI 10.1017/cem.2019.26 
the patients' initial treatment. ${ }^{9}$ PoCUS has the advantage of being immediately available and rapid to use, as well as being accurate in detecting decompensated heart failure in the emergency department (ED). ${ }^{8,10}$ Markarian et al. report on the reliability of a lung ultrasound score to independently predict severity of illness, as determined by the eventual admission to the intensive care unit or death, in elderly breathless ED patients. ${ }^{11}$ Lung ultrasound is being used increasingly in breathless patients, yet there are several ways to interpret the findings, many of which are based upon sonographic artefacts such as frequency of B-lines. The ability to use a defined score to triage the severity of illness in elderly breathless patients could be useful, in addition to any diagnostic benefits that PoCUS can add. Again, will this intervention impact outcome? It is difficult to say. Yet an early accurate prediction of disease severity and likely critical care disposition may help highlight the need for initial high dependency observation and guide resource allocation.

Finally, ever since clinicians picked up an ultrasound probe, there have been calls to ensure that these nonradiology providers adhere to high standards of clinical application and to patient safety issues such as infection control and cleanliness. Previous studies have reported variable levels of cleanliness when ultrasound probes used at the front line of care were inspected. Sanz et al. have previously reported that, although out of 110 samples, no isolates of methicillin-resistant Staphylococcus aureus were cultured, and that only one probe yielded a positive culture for methicillin-susceptible Staphylococcus aureus, ultrasound probes in the medicine, trauma, and pediatrics areas of their hospital were found to be clean only $65 \%, 33 \%$, and $70 \%$ of the time, respectively. ${ }^{12}$ Poonja et al. report their investigation into the contamination of ultrasound probes with residual blood, following use in trauma cases on the front line in the ED. ${ }^{13}$ They made daily assessments and assessments following Level 1 traumas, and out of 54 inspections found visible blood contamination on two separate occasions, both after traumas. There was no visible blood contamination on daily inspections. They also found occult blood on the probe on $10 \%$ of daily tests and $43 \%$ of post-trauma tests. Despite these concerning levels of contamination, there was an additional finding that subsequent cleaning with germicidal wipes removed all traces of occult blood during the study. Whether this also removes all traces of microbial contamination and results in an acceptable level of cleanliness for PoCUS equipment remains unclear. Further investigation is required to determine a standardized PoCUS transducer hygiene approach that not only minimizes risk of cross contamination, but also is workable in a busy ED.

We are encouraged by the continued PoCUS scholarship evident in this issue of CFEM and elsewhere, and we hope to see ongoing high-quality comparative research in this field. Front line clinicians should continue to be aware of the benefits and limitations of PoCUS, especially in critically ill patients, as they incorporate this tool into their clinical practice. They should also be aware of the potential for the transducer to provide not only a diagnosis, but also an unwanted contamination.

Keywords: Dyspnea, patient safety, PoCUS, shock

Competing interests: Dr. Atkinson and Dr. Lewis are co-directors or the Emergency Critical Care Ultrasound Course www. ECCUcourse.com, and are both editors of the textbook 'Point of Care Ultrasound for Emergency Medicine and Resuscitation'. Dr. Atkinson is Vice President, Ultrasound Research for the International Federation of Emergency Medicine.

\section{REFERENCES}

1. Moore CL, Copel JA. Point-of-care ultrasonography. N Engl 7 Med 2011;364(8):749-57.

2. Jones A, Tayal V, Sullivan D, et al. Randomized, controlled trial of immediate versus delayed goal-directed ultrasound to identify the cause of nontraumatic hypotension in emergency department patients. Crit Care Med 2004;32:1703-8.

3. Atkinson PR, McAuley DJ, Kendall RJ, et al. Abdominal and cardiac evaluation with sonography in shock (ACES): an approach by emergency physicians for the use of ultrasound in patients with undifferentiated hypotension. Emerg Med 7 2009;26:87-91.

4. Perera P, Mailhot T, Riley D, et al. The RUSH exam: rapid ultrasound in shock in the evaluation of the critically ill. Emerg Med Clin North Am 2010;28:29-56.

5. Stickles SP, Carpenter CR, Gekle R, et al. The diagnostic accuracy of a point-of-care ultrasound protocol for shock etiology: a systematic review and meta-analysis. CFEM 2019;21:406-17.

6. Atkinson P, Bowra J, Milne J, et al. International Federation for Emergency Medicine Consensus Statement: sonography in hypotension and cardiac arrest (SHoC): an international consensus on the use of point of care ultrasound for undifferentiated hypotension and during cardiac arrest. CFEM 2017;19(6):459-70.

7. Atkinson PR, Milne J, Diegelmann L, et al. Does point-of-care ultrasonography improve clinical outcomes in emergency department patients with undifferentiated hypotension? An international randomized controlled trial from the SHoC-ED investigators. Ann Emerg Med 2018;72 (4):478-89. 
8. Pivetta E, Goffi A, Nazerian P, et al. Lung ultrasound integrated with clinical assessment for the diagnosis of acute decompensated heart failure in the emergency department: a randomized controlled trial. Eur 7 Heart Fail 2019: doi: 10.1002/ejhf.1379. [Epub ahead of print]

9. Sartini S, Frizzi J, Borselli M, et al. Which method is best for an early accurate diagnosis of acute heart failure? Comparison between lung ultrasound, chest X-ray and NT pro-BNP performance: a prospective study. Intern Emerg Med 2017;12 (6):861-9.

10. McGivery K, Atkinson P, Lewis D, et al. Emergency department ultrasound for the detection of B-lines in the early diagnosis of acute decompensated heart failure: a systematic review and meta-analysis. CFEM 2018;20(3):343-52.

11. Markarian T, Zieleskiewicz L, Perrin G, et al. A lung ultrasound score for early triage of elderly patients with acute dyspnea. CFEM 2019;21(3):399-405.

12. Sanz GE, Theoret J, Liao MM, et al. Bacterial contamination and cleanliness of emergency department ultrasound probes. CFEM 2011;13(6):384-9.

13. Poonja Z, Uppal J, Netherton SJ, et al. Evaluation of emergency department ultrasound machines for the presence of occult blood. CFEM 2018;21(3):395-98. 